\title{
Role of DNMT3A, TET2, and IDH1/2 mutations in pre-leukemic stem cells in acute myeloid leukemia
}

\author{
Steven M. Chan · Ravindra Majeti
}

Received: 25 June 2013 / Accepted: 31 July 2013/Published online: 15 August 2013

(C) The Japanese Society of Hematology 2013

\begin{abstract}
Aberrant changes in the epigenome are now recognized to be important in driving the development of multiple human cancers including acute myeloid leukemia. Recent advances in sequencing technologies have led to the identification of recurrent mutations in genes that regulate DNA methylation including DNA methyltransferase 3A (DNMT3A), ten-eleven translocation 2 (TET2), and isocitrate dehydrogenase 1 (IDH1) and IDH2. These mutations have been shown to promote self-renewal and block differentiation of hematopoietic stem/progenitor cells. Acquisition of these mutations in hematopoietic stem cells can lead to their clonal expansion resulting in a pre-leukemic stem cell (pre-LSC) population. Pre-LSCs retain the ability to differentiate into the full spectrum of mature daughter cells but can become fully transformed with the acquisition of additional driver mutations. Here, we review the effects of mutations in DNMT3A, TET2, and IDH1/2 on mouse and human hematopoiesis, the current understanding of their role in pre-LSCs, and therapeutic strategies to eliminate this population which may serve as a cellular reservoir for relapse.
\end{abstract}

Keywords Leukemia stem cells - Acute myeloid leukemia $\cdot$ Epigenetics $\cdot$ DNMT3A $\cdot$ TET2 $\cdot$ IDH

\footnotetext{
S. M. Chan · R. Majeti

Department of Medicine, Division of Hematology, Cancer Institute, and Institute for Stem Cell Biology and Regenerative Medicine, Stanford University School of Medicine, Palo Alto, CA 94305, USA

\section{R. Majeti ( $\square)$}

Stanford Institute for Stem Cell Biology and Regenerative Medicine, Lokey Stem Cell Building, 265 Campus Drive, Stanford, CA 94305, USA

e-mail: rmajeti@stanford.edu
}

\section{Introduction}

Acute myeloid leukemia (AML) is a stem cell disease in which only a small fraction of leukemia cells possesses unlimited self-renewal capacity and the ability to serially transplant leukemia in immunodeficient mice [1]. The precise events that give rise to the founder leukemic stem cell (LSC) are unclear, but recent experimental evidence supports a model in which the step-wise accumulation of mutations in hematopoietic stem cells (HSC) and progenitor cells eventually leads to a fully transformed cell with impaired differentiation potential and unlimited selfrenewal capacity [2,3]. Pre-leukemic stem cells (pre-LSC) refer to the population of HSCs that harbor the early mutations, but not the entire complement of mutations necessary to become frank leukemia. Pre-LSCs by definition retain the ability to differentiate into the full spectrum of mature myeloid and lymphoid cells (Fig. 1).

Recent efforts in whole-genome and exome sequencing have greatly accelerated the identification of the underlying driver mutations in AML and other myeloid malignancies. Somatic mutations in genes that encode proteins involved in epigenetic regulation are now known to play a critical role in transformation [4]. Mutations of these epigenetic modifiers presumably lead to abnormal gene expression that ultimately promotes malignant transformation. The mechanism by which alterations in the epigenome contribute to the generation of pre-LSCs and LSCs is an area of intense investigation. A detailed understanding of these mechanisms may lead to novel therapies that selectively target LSCs as well as pre-LSCs which may contribute to relapse if not eradicated.

Epigenetic regulators that are recurrently mutated in AML can be classified into two main groups based on their primary target of action. The first group affects post- 


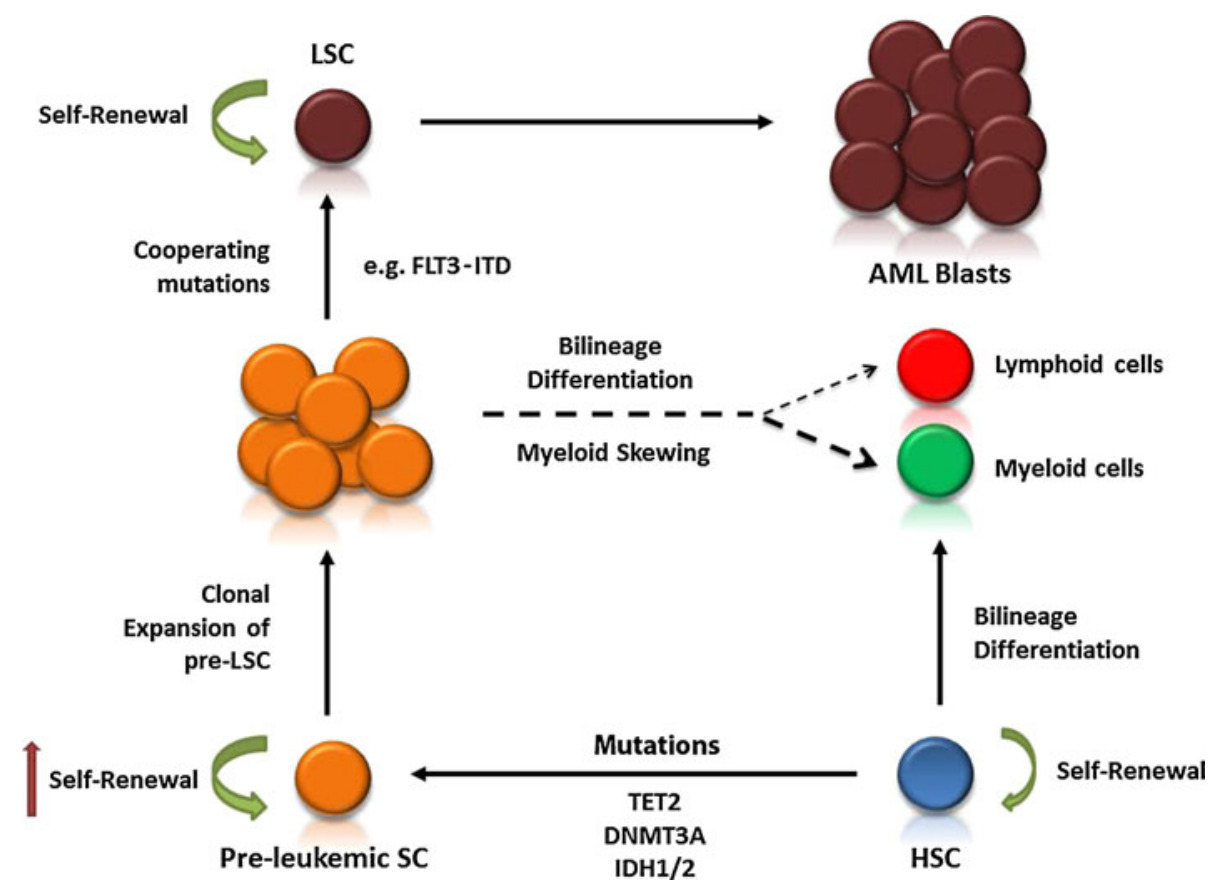

Fig. 1 Model of step-wise acquisition of mutations in the development of pre-leukemic stem cells (pre-LSCs) and fully transformed leukemic stem cells (LSCs) from hematopoietic stem cells (HSCs). Acquisition of mutations in genes involved in regulation of DNA methylation (TET2, DNMT3A, and IDH1/2) in HSCs leads to enhanced self-renewal and clonal expansion of a pre-LSC population. By definition, pre-LSCs do not cause overt disease and retain the ability to differentiate into lymphoid and myeloid lineages. However,

translational histone modifications and includes additional sex combs-like 1 (AXSL1), enhancer of zeste homologue 2 (EZH2), and mixed-lineage leukemia (MLL). The second group regulates DNA methylation of cytosines in the context of $\mathrm{CpG}$ dinucleotides and includes DNA methyltransferase 3A (DNMT3A) and ten-eleven translocation 2 (TET2). Mutations in isocitrate dehydrogenase 1 (IDH1) and IDH2 have been shown to affect both histone modifications and DNA methylation. Here, we review the current understanding of the effects of DNMT3A, TET2, and IDH1/2 mutations on hematopoiesis, their role in pre-LSCs in humans, and therapeutic strategies to target this population.

\section{DNMT3A mutations}

Methylation of cytosine at the 5-position [5-methyl cytosine $(5 \mathrm{mC})]$ in the context of $\mathrm{CpG}$ dinucleotides is generally associated with a transcriptionally silent chromatin configuration often referred to as heterochromatin [5]. The family of DNA methyltransferases that catalyzes this modification is comprised of three members: DNMT1, DNMT3A, and DNMT3B. DNMT1 is primarily a maintenance enzyme that copies the methylation marks of they may skew towards myeloid development in some circumstances (e.g. TET2 mutations). Acquisition of additional cooperating mutations (e.g. FLT3-ITD) converts a pre-LSC into a fully transformed LSC with unlimited self-renewal potential and impaired differentiation. The eradication of both pre-LSCs and LSCs may be required to prevent disease relapse as both populations may serve as a cellular reservoir for relapse

hemimethylated DNA after replication [6]. Mutations in DNMT1 have been reported in colorectal cancer [7]. Recently, the Cancer Genome Atlas (TCGA) Research Network reported a DNMT1 mutation in one AML sample [8]. Whether mutations in DNMT1 are recurrent and play a role in driving leukemogenesis requires further investigation. In contrast to DNMT1, DNMT3A and DNMT3B are able to act on unmethylated DNA substrates and are referred to as de novo methyltransferases [5] (Fig. 2). A somatic mutation in DNMT3A was initially discovered by Ley and colleagues [9] by whole-genome sequencing of an AML sample with normal karyotype. They subsequently found that $22 \%$ of de novo AML cases harbored mutations in DNMT3A, which are enriched in patients with intermediate-risk cytogenetics [9]. Mutations in DNMT3A have also been reported in about $10 \%$ of patients with myelodysplastic syndrome (MDS) [10] and myeloproliferative neoplasms (MPN) [11]. Although a variety of missense, nonsense, frame-shift, and splice-site mutations were found along the gene, the most common mutation was located at a single amino acid residue (arginine 882) [9]. Mutant enzymes with an amino acid substitution at this location have been shown to have reduced DNA methylation activity [12]. The remaining mutations generally result in 

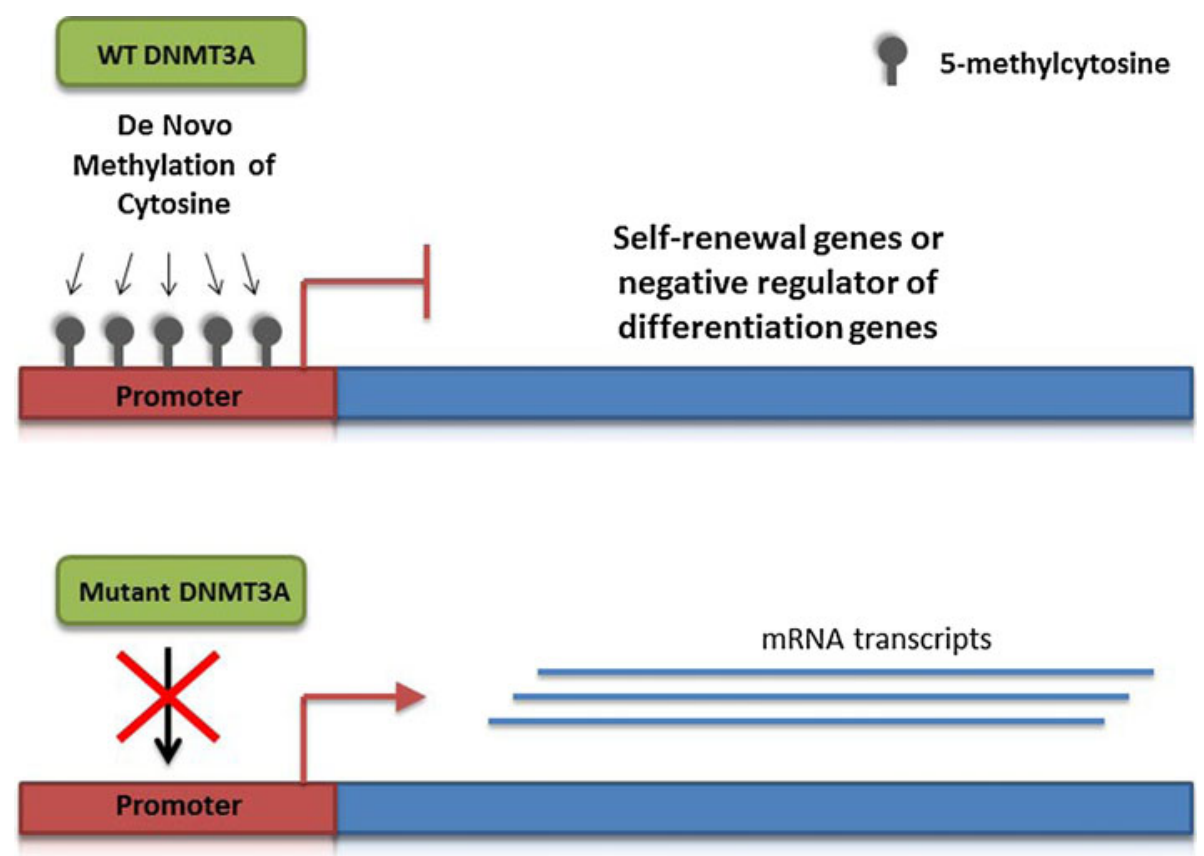

Fig. 2 Mechanism of dysregulated gene expression induced by DNMT3A mutations. DNMT3A catalyzes de novo methylation of DNA at the 5-position of cytosine in the context of $\mathrm{CpG}$ dinucleotides. DNA methylation is generally associated with transcriptional repression and can suppress the expression of self-renewal genes or genes that negatively regulate differentiation. DNMT3A activity

the premature truncation of the protein product. Therefore, mutations in DNMT3A appear to disrupt the function of the enzyme.

To investigate the effect of mutant DNMT3A in a cell line, Yan and colleagues [12] overexpressed DNMT3A Arg882 mutants in 32D cells (an interleukin-3 dependent murine myeloid cell line) and found increased cell proliferation even in the absence of IL-3. The mechanism by which the mutant enzyme conferred a proliferative advantage and growth factor independence was not investigated in this study. To examine the effects of DNMT3A deficiency on hematopoiesis in vivo, Challen and colleagues [13] generated inducible conditional DNMT3A knock-out mice and transplanted purified HSCs (defined as

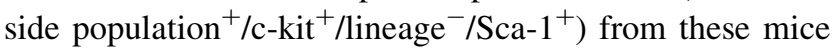
along with competing wild-type control HSCs into lethally irradiated wild-type recipients. The effect of DNMT3A deficiency on the HSC contribution to peripheral blood cell generation was not apparent in primary recipients, but with subsequent serial transplantations there was a progressive increase in peripheral blood cell generation from DNMT3A-deficient HSCs compared with wild-type HSCs. Examination of the bone marrow revealed a striking expansion of the HSC compartment that was not associated with increased cell cycling or resistance to apoptosis. Importantly, the degree of HSC expansion was out of appears to be necessary for normal hematopoietic stem cell (HSC) differentiation. Mutations in DNMT3A that disrupt enzymatic activity may promote self-renewal and block differentiation of HSCs by modifying DNA methylation at gene regulatory regions and thereby increasing the transcription of self-renewal genes and negative regulators of differentiation

proportion to the increase in peripheral blood contribution, indicative of impairment in differentiation. It is worth noting that none of the recipients of DNMT3A-null HSCs developed overt myeloproliferative disease indicating that additional collaborating mutations are required for development of disease.

The global $5 \mathrm{mC}$ level in HSCs was unaffected by DNMT3A ablation, but analysis at specific genomic regions identified sites of hypomethylation and unexpectedly, hypermethylation [13]. The genes associated with hypomethylation were found to be frequently overexpressed in leukemias. Furthermore, global transcriptional profiling of DNMT3A-deficient HSCs showed upregulation of genes that were previously reported to be highly expressed in normal HSCs (e.g. Gata3, Runxl) and down-regulation of genes involved in differentiation (e.g. Ikaros, PU.l). Intriguingly, there was a poor correlation between differential DNA methylation and gene expression.

An important caveat of this mouse model is that the complete deletion of both copies of DNMT3A may not recapitulate the biology of the heterozygous mutations found in the vast majority of AML cases [9]. Furthermore, recurrent mutations at a single amino acid position generally suggest a gain-of-function mechanism or the acquisition of a neomorphic activity. Although there is currently no experimental evidence to support this, heterozygous 
knock-in mouse models are required to fully address these possibilities.

Taken together, this study provides experimental evidence that complete loss of DNMT3A activity results in enhanced self-renewal of HSCs and a block in their differentiation. The findings support a model in which acquisition of a DNMT3A mutation in an HSC promotes its expansion into a pre-LSC clone by increasing selfrenewal (Fig. 1). The observation that all the cells in the bone marrow of MDS patients with DNMT3A mutations contain the mutation regardless of their blast count is consistent with this model [10]. DNMT3A mutations have also been shown to be remarkably stable in disease evolution [14-16] which may be due to their persistence in the pre-LSC compartment. As such, therapies that eradicate pre-LSCs harboring DNMT3A mutations may be particularly useful in the prevention of relapse. A recent provocative study reported that decitabine was associated with complete response (CR) rate of $75 \%$ in patients with DNMT3A mutations as compared with a CR rate of $34 \%$ in patients with wild-type DNMT3A [17]. Although the number of patients in this study was small, the results suggest that hypomethylating agents may be effective in targeting pre-LSCs harboring DNMT3A mutations.

\section{TET2 mutations}

The TET family of enzymes is composed of three members (TET1, TET2, and TET3). They are Fe(II)- and $\alpha$-ketoglutarate-dependent enzymes that catalyze the oxidation of $5 \mathrm{mC}$ to 5-hydroxymethylcytosine (5-hmC) [18]. 5-hmC appears to be an intermediate product in DNA demethylation mechanisms. Passive demethylation occurs when DNMT1, which does not recognize 5-hmC, fails to copy methylation patterns during DNA replication [5, 19]. An active process that requires conversion of 5 -hmC to other intermediates (5-formylcytosine and 5-carboxycytosine) followed by base excision repair pathway processing has also been described [20, 21] (Fig. 3).

TET1 was originally discovered as the fusion partner of MLL in AML patients with the $\mathrm{t}(10 ; 11)(\mathrm{q} 22 ; \mathrm{q} 23)$ [22]. TET2 was later identified to be deleted or mutated in diverse myeloid malignancies including AML, MDS, MPN, chronic myelomonocytic leukemia (CMML), and systemic mastocytosis [23-25]. The overall frequency of TET2 mutations is about 10-20\% in AML [23] and as high as $50 \%$ in CMML patients [25]. TET2 mutations include deletions, nonsense mutations, and missense mutations at highly conserved residues and are predicted to inactivate the function of the enzyme [23]. The vast majority of patients have heterozygous mutations indicating that haploinsufficiency of TET2 is sufficient to drive leukemogenesis.
Since the discovery of TET2 mutations, a number of TET2 knock-out mouse models have been created to delineate its role in hematopoiesis [26-31]. The mouse models consistently showed an expansion of the hematopoietic stem/progenitor compartment in association with increased self-renewal. Disruption of TET2 also resulted in the skewing of differentiation to the granulomonocytic lineages. Knockdown of TET2 with shRNA lentiviral vectors in $\mathrm{CD}_{3} 4^{+}$human cord blood progenitor cells also skewed differentiation toward the granulomonocytic lineage at the expense of lymphoid and erythroid lineages ex vivo [32]. In some of the mouse models, an overt myeloproliferative disease characterized by splenomegaly, monocytosis, and extramedullary hematopoiesis developed following TET2 deletion after a latency period of about 3-6 months [26]. The latency period suggests that the acquisition of additional collaborating mutations is required for the development of disease, and that the acquisition of TET2 mutations is an early event in the stepwise progression from HSC to myeloid malignancy.

Several lines of evidence now support the model that TET2 inactivation is an early event in leukemogenesis and results in clonal expansion of pre-LSCs in humans. Initial genotyping studies of colonies derived from single $\mathrm{CD} 34^{+} \mathrm{CD} 38^{-}$cells of MPN patients with both JAK2 V617F and TET2 mutations showed that the JAK2 mutation was not observed in the absence of TET2 mutations [23]. This observation suggests that the JAK2 mutation was acquired after the occurrence of TET2 mutations. In another study, Busque et al. [33] investigated the genetic basis of age-associated skewing (AAS) which refers to deviation from the 1:1 X-chromosome inactivation ratio observed in normal female mammalian cells over time. The skewing is most pronounced in the myeloid compartment. They hypothesized that acquisition of somatic mutations that confer enhanced self-renewal in HSCs leads to their clonal expansion and skewing of the X-chromosome inactivation ratio. They initially identified a missense mutation in TET2 in an elderly woman with AAS, along with mutations in 4 other genes including DNMT3A. They subsequently sequenced the 5 genes in a larger number of samples with and without AAS. TET2 mutations were found in $5.6 \%$ of the elderly women with AAS. The mutations were found in the DNA from granulocytes but not from lymphocytes, which is consistent with the granulomonocytic skewing of HSC differentiation observed in mouse models. An alternative explanation is that a myeloid-committed progenitor, not an HSC, was the initial cell that acquired the TET 2 mutation, which led to its persistence and expansion through enhanced self-renewal capacity. Importantly, the individuals with TET2 mutations did not have overt hematologic disorders. Results from this study are consistent with the model in which disruption of 
Fig. 3 Mechanism of dysregulated gene expression induced by TET 2 and IDH $1 / 2$ mutations. a TET 2 catalyzes the hydroxylation of

5-methylcytosine $(5-\mathrm{mC})$ in DNA resulting in the formation of 5-hydroxymethylcytosine (5hmC). $\alpha$-ketoglutarate $(\alpha-K G)$ is an essential cofactor in this reaction. 5-hmC appears to be an intermediate product in the DNA demethylation pathway which involves enzymes in the base excision repair (BER) pathway. TET2-mediated DNA demethylation may be necessary for the expression of genes that promote differentiation and genes that suppress self-renewal in normal hematopoiesis.

b Mutations that disrupt TET2 activity impair hydroxylation of 5-mC and consequently DNA demethylation at gene regulatory regions. This can result in transcriptional downregulation of genes that promote differentiation and genes that negatively regulate selfrenewal. Mutant IDH1 or IDH2 gain a neomorphic activity that catalyzes the conversion of $\alpha-K G$ to the oncometabolite, D-2-hydroxyglutarate (D-2-HG). D-2-HG inhibits the activity of $\alpha$-KG-dependent dioxygenases including TET2, which leads to changes in DNA methylation and gene expression similar to those seen with TET 2 mutations

\section{A}

\section{WT TET2}

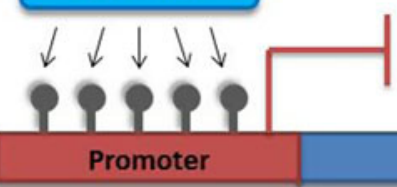

a-KG $\downarrow$ Hydroxylation of 5-methylcytosine

९९९९९

Promoter

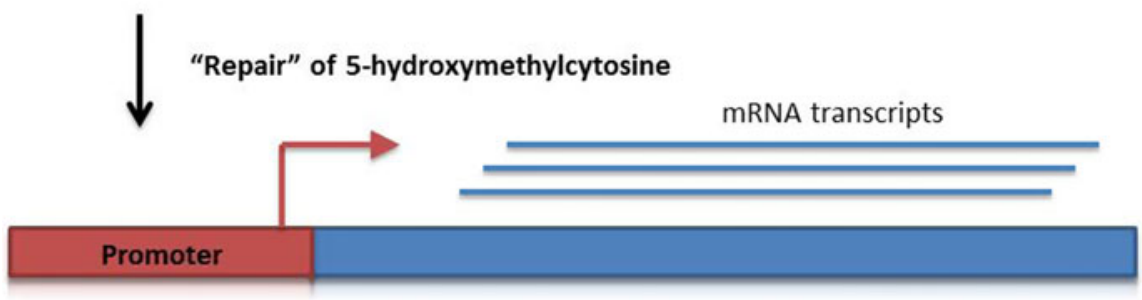

B

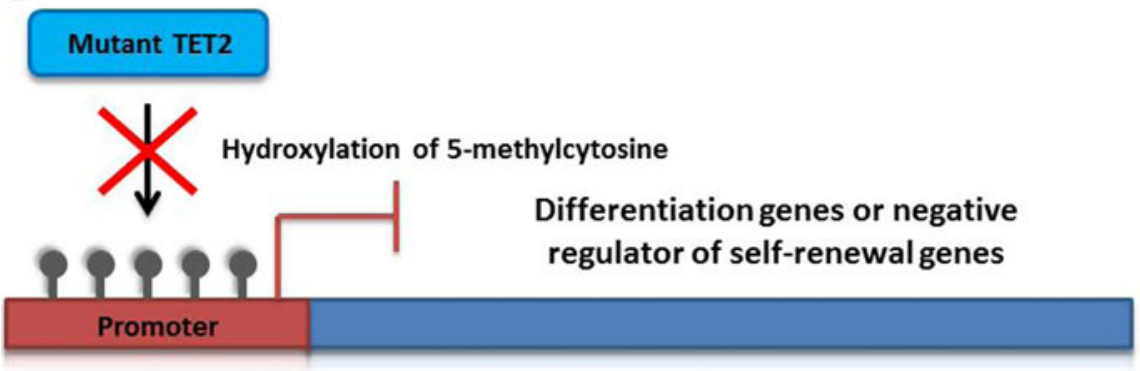

5-methylcytosine

5-hydroxymethylcytosine

\section{Differentiation genes or negative} regulator of self-renewal genes

osine

D-2-HG

WT TET2

$\mathrm{O}_{\mathrm{OH}}^{\mathrm{O}}$

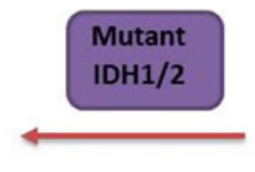

$\alpha-K G$
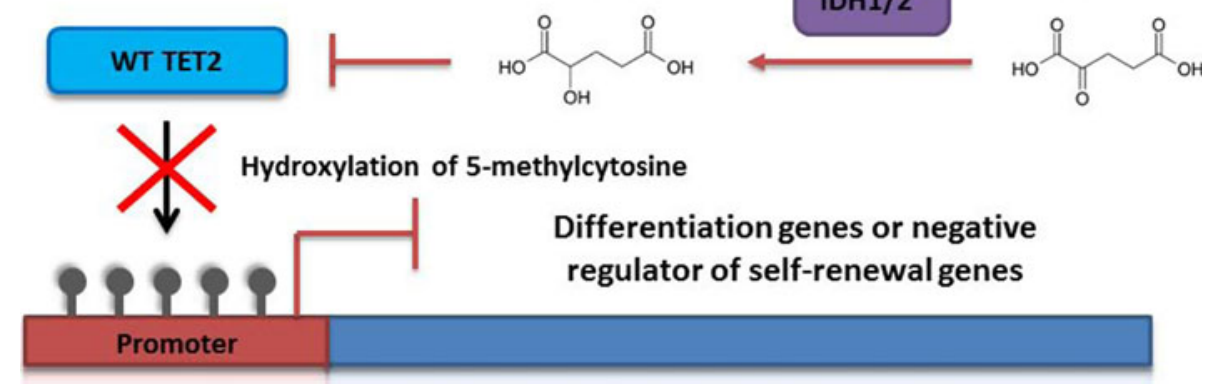

TET2 results in expansion of a clonal pre-LSC population prior to the onset of myeloid malignancy (Fig. 1).

A recent study from our laboratory provides additional evidence that TET2 inactivation results in clonal expansion of HSCs in humans and is an early event in AML leukemogenesis [34]. Our earlier work demonstrated that HSCs with the ability to engraft immunodeficient NOD/SCID/ IL2R $\gamma$-null mice and differentiate into lymphoid and myeloid lineages are present in hematopoietic tissues of AML patients [35]. These residual HSCs can be prospectively purified from LSCs based on differences in their surface immunophenotype. We found that residual HSCs, 
as defined by their ability to support bilineage engraftment, were predominantly in the lineage ${ }^{-} \mathrm{CD} 34^{+} \mathrm{CD}^{-} 8^{-} \mathrm{TIM}^{-}$ $\mathrm{CD}^{-} 9^{-}$population. To investigate the serial acquisition of mutations in HSCs of AML patients, we reconstructed the history of events by genotyping individual colonies expanded from single residual HSCs for mutations found in the leukemic blast population. In the two patients with biallelic mutations in TET2, we found that acquisition of TET2 mutations were early events because the vast majority of residual HSCs carried either one or both TET2 mutations. In contrast, FLT3 mutations were only detected in frankly leukemic cells, but not in residual HSCs. Furthermore, residual HSC clones harboring biallelic TET2 mutations were more numerous than clones with only one mutation, and the TET2 heterozygous population was in turn more numerous than HSCs lacking all mutations. Taken together, our observations provide the most direct evidence that disruption of TET2 activity confers enhanced self-renewal in HSCs resulting in a competitive advantage over non-mutated cells and expansion of the mutated clone over time. Importantly, this pre-LSC population containing TET2 mutations may serve as a reservoir for relapse if it is not eradicated with AML therapy. This hypothesis is supported by results of a recent study that compared the mutation profiles of 34 AML patients at diagnosis and at relapse [15]. The study showed that TET2 mutations were stable in the 4 patients who carried the mutation. However, the number of patients in this study was small and this pattern was not seen in another study with a larger number of paired samples that reported frequent loss of the original TET2 mutations at relapse [36]. Discrepancies between the two studies may be related to differences in the sensitivity of the detection assays employed. However, it is worth noting that in the latter study, all the patients that had detectable TET2 mutations at the time of morphologic complete remission eventually relapsed. Whether the TET2 mutations resided in residual HSCs, or in a small population of LSCs, is not clear. Addressing this issue will require genotyping of residual HSCs from remission samples. Nevertheless, this observation supports the possibility that at least in some cases, pre-LSCs with TET2 mutations may contribute to relapsed disease and will need to be targeted by AML therapies for prevention of relapse. Notably, the presence of TET2 mutations has been associated with an $82 \%$ response rate to azacitidine [37]. The use of hypomethylating agents to target the TET2-mutated pre-LSC population should be evaluated in future studies.

\section{IDH1/2 mutations}

IDH1 and IDH2 are enzymes in the citric acid cycle that catalyze the oxidative decarboxylation of isocitrate, producing $\alpha$-ketoglutarate $(\alpha-K G)$ and NADPH in the process. A mutation in the IDH1 gene was identified by whole-genome sequencing of an AML sample with normal karyotype in 2009 [38]. This and other studies confirmed that the mutation is recurrent and occurs not only in the cytosolic IDH1 enzyme but also in IDH2, the mitochondrial homolog of IDH1 [38-42]. Overall, about $15 \%$ of AML patients have mutations in either IDH1 or IDH2 and the mutations are enriched in patients with normal cytogenetics [43]. Mutations in IDH1/2 have also been found in other myeloid malignancies including MDS and MPN at lower frequencies [44, 45]. IDH mutations are highly frequent (80-90\%) in low-grade malignant gliomas [39, 42]. A growing number of cancers have since been found to harbor IDH mutations at varying frequencies including cholangiocarcinomas, chondrosarcomas, and angioimmunoblastic T-cell lymphomas [46-48].

In almost all cases, IDH mutations are heterozygous and occur at three highly conserved arginine residues: IDH1 R132, IDH2 R140 and IDH2 R172 [49]. These findings are consistent with the mutations conferring a gain-of-function phenotype. An important clue to the mechanism by which IDH mutations cause malignant transformation came from the observation that TET2 mutations are mutually exclusive with IDH mutations in the vast majority of patients $[50,51]$. Mutual exclusivity suggests that the two mutations are acting on the same pathways. The connection between the two mutations became apparent when the mutant IDH enzymes were shown to possess a neomorphic activity that catalyzes the conversion of $\alpha-K G$ to D-2-hydroxyglutarate (D-2-HG), consuming NADPH in the process $[40,41,52]$. D-2-HG is normally present at very low levels in non-mutated cells, but accumulates to over 100-fold higher levels in IDH-mutated AML and glioma cells $[40,52]$. D-2-HG is structurally similar to $\alpha-K G$ and was found to be a competitive inhibitor of multiple $\alpha-\mathrm{KG}$ dependent dioxygenases including TET2 [50, 53]. In line with these studies, overexpression of mutant IDH1 was found to inhibit the hydroxylation of $5 \mathrm{mC}$ by $\mathrm{TET} 2$ in 293 T cells [50]. Consistent with the role of 5-hmC in the DNA demethylation process, IDH-mutated samples were found to have a specific hypermethylation signature similar to that of TET2-mutated samples, and $93 \%$ of the genes aberrantly expressed in TET2-mutated AMLs were also aberrantly expressed in IDH-mutated AMLs [50]. Furthermore, a recent study showed that TET2 knockdown recapitulated the ability of mutant IDH1 and D-2-HG to promote transformation of an erythroleukemia cell line (TF-1) [54]. Taken together, the inhibition of TET2 activity by $\mathrm{D}-2-\mathrm{HG}$ produced by mutant IDH enzymes plays a critical role in driving leukemogenesis (Fig. 3).

Several lines of evidence suggest that other $\alpha-K G-$ dependent dioxygenases may also play a role in 
transformation of IDH-mutated cells. In vitro studies initially showed that D-2-HG can inhibit Jumonji-C domain containing histone demethylases (JHDMs) [53, 55]. Accordingly, overexpression of mutant IDH in immortalized astrocytes and 3T3-L1 cells resulted in a progressive increase in global histone methylation, in particular $\mathrm{H} 3 \mathrm{~K} 9$ methylation, which is a repressive mark. The increase in histone methylation was associated with a block in differentiation in 3T3-L1 cells [56]. EglN prolyl hydroxylases are $\alpha$-KG-dependent dioxygenases that mark hypoxia inducible factor- $1 \alpha$ (HIF-1 $\alpha$ ) for proteasome-mediated degradation. Surprisingly, D-2-HG was found to be an agonist of EglN activity leading to diminished levels of HIF- $1 \alpha$ in some cellular contexts [57]. The decrease in HIF- $1 \alpha$ activity contributed to the transformation of astrocytes in vitro. In contrast to TET2, the role of JHDM inhibition and EglN activation by D-2-HG in the transformation of hematopoietic cells is less clear and requires further investigation.

Initial studies investigating the effects of mutant IDH on myeloid differentiation showed that stable expression of mutant IDH2 in 32D cells increased the surface expression of c-kit, which is more highly expressed in immature progenitor cells [50]. Knockdown of TET2 expression also led to an in increase in c-kit expression. Similarly, expression of IDH2 R140Q in ex vivo cultures of murine bone marrow cells impaired myeloid differentiation as determined by $\mathrm{Gr}-1 / \mathrm{Mac}-1$ expression and increased the proportion of lineage ${ }^{-} / \mathrm{Sca}^{+} / \mathrm{cKit}^{+}$(LSK) cells [50].

To investigate the effects of IDH mutations on hematopoiesis in vivo, Sasaki and colleagues [58] used the loxstop-lox system to generate conditional knock-in (KI) mice, in which IDH1 R132H was inserted into the endogenous Idhl locus. The mutant gene was expressed specifically in cells of the myeloid lineage with the LysM promoter driving Cre expression. The proportion of LSK cells in the bone marrow expanded by about fivefold in 42-46-week-old LysM-KI mice, but the proportion of CMPs, GMPs, MEPs, and CLPs in the bone marrow was not affected. Although the bone marrow cells from LysMKI mice had a higher serial plating capacity than control cells, they did not have an advantage over wild-type cells in competitive repopulation transplant assays. The hematologic parameters of LysM-KI mice were remarkably normal, except for a modest decrease in hemoglobin in older mice. The mice developed splenomegaly over time with histologic evidence of extramedullary hematopoiesis, but their lifespans were normal. Progression to acute leukemia was not reported, indicating that additional collaborating mutations are required. Thus far, this study is the only mouse model of IDH mutation in the hematopoietic system. Although there was clear evidence of expansion of the hematopoietic stem/progenitor compartment, the magnitude of the effect was small in comparison with TET2-deficient mouse models. The difference in phenotype may be due to less effective inhibition of TET2 activity in IDH1-mutated cells compared with loss-offunction TET2 mutations, and/or the involvement of other pro-oncogenic pathways (e.g. inhibition of histone demethylases and HIF-1 $\alpha$ signaling) in IDH1-mutated cells that would not be expected to occur in TET2-mutated cells. In addition, mutant IDH expression and D-2-HG accumulation can have a negative effect on cell proliferation in some cellular contexts $[54,59]$, which may explain the less robust expansion of IDH1-mutated hematopoietic stem/ progenitor cells.

The expansion of LSK cells in IDH1-mutant mice suggests that the acquisition of IDH mutations may confer enhanced self-renewal in HSCs leading to their clonal expansion over time (Fig. 1). The presence of IDH mutations in pre-LSCs is supported by the persistence of these mutations in AML patients in long-term complete remission following induction chemotherapy [60]. In addition, IDH1 mutations appear to be stable during disease evolution. Paired analysis of IDH1-mutated samples showed that the same mutations were present at both diagnosis and relapse in 11 patients [61]. Mutations in IDH2 were also found to be stable during disease evolution [62]. In our recently published study of clonal evolution in pre-leukemic HSCs, there was one sample with IDH1 mutation, but this mutation was not found in sorted residual HSCs by our deep sequencing assay which has a lower threshold of sensitivity of $<1 \%$ variant allele [34]. There are several possible explanations for this finding. The first is that the size of the IDH1-mutated residual HSC clone was simply below the sensitivity of our assay. The second is that the acquisition of IDH1 mutations may be a late event in leukemogenesis analogous to FLT3 mutations. Another possibility is that the IDH1 mutation could have resided in a more mature progenitor cell population that evaded our sorting strategy for residual HSCs. Analysis of a larger population of IDH1/2-mutated patients will be required to better delineate the role of this mutation in the evolution of pre-LSCs.

Unlike TET2 and DNMT3A mutations, IDH mutations confer a neomorphic gain-of-function to the enzyme. This represents a unique therapeutic opportunity because inhibitors can be designed to specifically inhibit the mutant enzymes. Importantly, a recent study showed that D-2-HG is sufficient to promote growth factor independence and block erythropoietin (EPO)-induced differentiation in TF-1 cells, and that the effects are reversible [54]. The development of specific mutant IDH1/2 inhibitors and their effects on AML cells have since been reported [54, 63, 64]. Exposure of TF-1 cells to EPO normally results in erythroid differentiation which can be monitored by increased 
expression of glycophorin A, hemoglobin, and the transcription factor, KLF1. Expression of mutant IDH1/2 or exposure to D-2-HG blocks this response to EPO [54, 63]. Treatment of IDH1 R132H expressing TF-1 cells with a mutant IDH1 specific inhibitor (AGX-891) at least partially reversed the differentiation block [54]. A similar effect was observed using a mutant IDH2 specific inhibitor (AGI6780) on IDH2 R140Q expressing TF-1 cells [63]. The mutant IDH2 inhibitor also increased the expression of myeloid differentiation markers (CD11b, CD14, CD15, myeloperoxidase) on ex vivo-cultured primary AML cells harboring the IDH2 R140Q mutation, although the effect and the number of samples tested were small [63]. Whether these effects can be reproduced in vivo requires further investigation. Nevertheless, these inhibitors have the potential to eliminate LSCs, as well as pre-LSCs, by limiting their self-renewal and promoting their differentiation in vivo. Clinical trials involving the use of these compounds in patients with AML and other malignancies with IDH mutations will provide important insights into their potential therapeutic value.

\section{Conclusions}

The identification of recurrent mutations in genes that regulate DNA methylation in AML has fundamentally transformed our understanding of the basis of the disease. The generation of mouse models of these mutations has begun to unravel the mechanisms by which these mutations drive leukemogenesis. However, the impact of these mutations on pre-LSCs is still largely unexplored and speculative at this point. Furthermore, the effectiveness of current therapies on the elimination of pre-LSCs is unknown. Studying the role of these mutations in pre-LSCs in primary human samples and the impact of targeted therapies on this population is an important step towards the development of more effective AML therapies.

Acknowledgments S.M.C is supported by the Stanford Division of Hematology. R.M. holds a Career Award for Medical Scientists from the Burroughs Wellcome Fund and is a New York Stem Cell Foundation Robertson Investigator. The authors report no competing interests.

\section{References}

1. Hope KJ, Jin L, Dick JE. Acute myeloid leukemia originates from a hierarchy of leukemic stem cell classes that differ in selfrenewal capacity. Nat Immunol. 2004;5:738-43.

2. Jan M, Majeti R. Clonal evolution of acute leukemia genomes. Oncogene. 2013;32:135-40.

3. Pandolfi A, Barreyro L, Steidl U. Concise review: preleukemic stem cells: molecular biology and clinical implications of the precursors to leukemia stem cells. Stem Cells Transl Med. 2013;2:143-50.

4. Shih AH, Abdel-Wahab O, Patel JP, Levine RL. The role of mutations in epigenetic regulators in myeloid malignancies. Nat Rev Cancer. 2012;12:599-612.

5. Butler JS, Dent SY. The role of chromatin modifiers in normal and malignant hematopoiesis. Blood. 2013;121:3076-84.

6. Song J, Rechkoblit O, Bestor TH, Patel DJ. Structure of DNMT1DNA complex reveals a role for autoinhibition in maintenance DNA methylation. Science. 2011;331:1036-40.

7. Kanai Y, Ushijima S, Nakanishi Y, Sakamoto M, Hirohashi S. Mutation of the DNA methyltransferase (DNMT) 1 gene in human colorectal cancers. Cancer Lett. 2003;192:75-82.

8. Cancer Genome Atlas Research Network. Genomic and epigenomic landscapes of adult de novo acute myeloid leukemia. New Engl J Med. 2013;368:2059-74.

9. Ley TJ, Ding L, Walter MJ, McLellan MD, Lamprecht T, Larson DE, et al. DNMT3A mutations in acute myeloid leukemia. New Engl J Med. 2010;363:2424-33.

10. Walter MJ, Ding L, Shen D, Shao J, Grillot M, McLellan M, et al. Recurrent DNMT3A mutations in patients with myelodysplastic syndromes. Leukemia. 2011;25:1153-8.

11. Stegelmann F, Bullinger L, Schlenk RF, Paschka P, Griesshammer M, Blersch C, et al. DNMT3A mutations in myeloproliferative neoplasms. Leukemia. 2011;25:1217-9.

12. Yan XJ, Xu J, Gu ZH, Pan CM, Lu G, Shen Y, et al. Exome sequencing identifies somatic mutations of DNA methyltransferase gene DNMT3A in acute monocytic leukemia. Nat Genet. 2011;43:309-15.

13. Challen GA, Sun D, Jeong M, Luo M, Jelinek J, Berg JS, et al. Dnmt3a is essential for hematopoietic stem cell differentiation. Nat Genet. 2012;44:23-31.

14. Hou HA, Kuo YY, Liu CY, Chou WC, Lee MC, Chen CY, et al. DNMT3A mutations in acute myeloid leukemia: stability during disease evolution and clinical implications. Blood. 2012;119: 559-68.

15. Wakita S, Yamaguchi H, Omori I, Terada K, Ueda T, Manabe E, et al. Mutations of the epigenetics-modifying gene (DNMT3a, TET2, IDH1/2) at diagnosis may induce FLT3-ITD at relapse in de novo acute myeloid leukemia. Leukemia. 2012;27(5):1044-52.

16. Kronke J, Bullinger L, Teleanu V, Tschurtz F, Gaidzik VI, Kuhn MW, et al. Clonal evolution in relapsed NPM1 mutated acute myeloid leukemia. Blood. 2013;122(1):100-8.

17. Metzeler KH, Walker A, Geyer S, Garzon R, Klisovic RB, Bloomfield CD, et al. DNMT3A mutations and response to the hypomethylating agent decitabine in acute myeloid leukemia. Leukemia. 2012;26:1106-7.

18. Ko M, Huang Y, Jankowska AM, Pape UJ, Tahiliani M, Bandukwala HS, et al. Impaired hydroxylation of 5-methylcytosine in myeloid cancers with mutant TET2. Nature. 2010;468:839-43.

19. Valinluck V, Sowers LC. Endogenous cytosine damage products alter the site selectivity of human DNA maintenance methyltransferase DNMT1. Cancer Res. 2007;67:946-50.

20. Ito S, Shen L, Dai Q, Wu SC, Collins LB, Swenberg JA, et al. Tet proteins can convert 5-methylcytosine to 5-formylcytosine and 5-carboxylcytosine. Science. 2011;333:1300-3.

21. He YF, Li BZ, Li Z, Liu P, Wang Y, Tang Q, et al. Tet-mediated formation of 5-carboxylcytosine and its excision by TDG in mammalian DNA. Science. 2011;333:1303-7.

22. Ono R, Taki T, Taketani T, Taniwaki M, Kobayashi H, Hayashi Y. LCX, leukemia-associated protein with a CXXC domain, is fused to MLL in acute myeloid leukemia with trilineage dysplasia having $\mathrm{t}(10 ; 11)(\mathrm{q} 22 ; \mathrm{q} 23)$. Cancer Res. 2002;62:4075-80.

23. Delhommeau F, Dupont S, Della Valle V, James C, Trannoy S, Masse A, et al. Mutation in TET2 in myeloid cancers. New Engl J Med. 2009;360:2289-301. 
24. Tefferi A, Levine RL, Lim KH, Abdel-Wahab O, Lasho TL, Patel $\mathrm{J}$, et al. Frequent TET2 mutations in systemic mastocytosis: clinical, KITD816V and FIP1L1-PDGFRA correlates. Leukemia. 2009;23:900-4.

25. Kosmider O, Gelsi-Boyer V, Ciudad M, Racoeur C, Jooste V, Vey $\mathrm{N}$, et al. TET2 gene mutation is a frequent and adverse event in chronic myelomonocytic leukemia. Haematologica. 2009;94: 1676-81.

26. Moran-Crusio K, Reavie L, Shih A, Abdel-Wahab O, NdiayeLobry D, Lobry C, et al. Tet2 loss leads to increased hematopoietic stem cell self-renewal and myeloid transformation. Cancer Cell. 2011;20:11-24.

27. Quivoron C, Couronne L, Della Valle V, Lopez CK, Plo I, Wagner-Ballon $\mathrm{O}$, et al. TET2 inactivation results in pleiotropic hematopoietic abnormalities in mouse and is a recurrent event during human lymphomagenesis. Cancer Cell. 2011;20:25-38.

28. Ko M, Bandukwala HS, An J, Lamperti ED, Thompson EC, Hastie R, et al. Ten-Eleven-Translocation 2 (TET2) negatively regulates homeostasis and differentiation of hematopoietic stem cells in mice. Proc Natl Acad Sci USA. 2011;108:14566-71.

29. Kunimoto H, Fukuchi Y, Sakurai M, Sadahira K, Ikeda Y, Okamoto S, et al. Tet2 disruption leads to enhanced self-renewal and altered differentiation of fetal liver hematopoietic stem cells. Sci Rep. 2012;2:273.

30. Shide K, Kameda T, Shimoda H, Yamaji T, Abe H, Kamiunten A, et al. TET2 is essential for survival and hematopoietic stem cell homeostasis. Leukemia. 2012;26:2216-23.

31. Li Z, Cai X, Cai CL, Wang J, Zhang W, Petersen BE, et al. Deletion of Tet 2 in mice leads to dysregulated hematopoietic stem cells and subsequent development of myeloid malignancies. Blood. 2011;118:4509-18.

32. Pronier E, Almire C, Mokrani H, Vasanthakumar A, Simon A, da Costa Reis Monte Mor B, et al. Inhibition of TET2-mediated conversion of 5-methylcytosine to 5-hydroxymethylcytosine disturbs erythroid and granulomonocytic differentiation of human hematopoietic progenitors. Blood. 2011;118:2551-5.

33. Busque L, Patel JP, Figueroa ME, Vasanthakumar A, Provost S, Hamilou Z, et al. Recurrent somatic TET2 mutations in normal elderly individuals with clonal hematopoiesis. Nat Genet. 2012;44:1179-81.

34. Jan M, Snyder TM, Corces-Zimmerman MR, Vyas P, Weissman IL, Quake SR, et al. Clonal evolution of preleukemic hematopoietic stem cells precedes human acute myeloid leukemia. Sci Transl Med. 2012;4:149ra18.

35. Jan M, Chao MP, Cha AC, Alizadeh AA, Gentles AJ, Weissman IL, et al. Prospective separation of normal and leukemic stem cells based on differential expression of TIM3, a human acute myeloid leukemia stem cell marker. Proc Natl Acad Sci USA. 2011;108:5009-14.

36. Chou WC, Chou SC, Liu CY, Chen CY, Hou HA, Kuo YY, et al. TET2 mutation is an unfavorable prognostic factor in acute myeloid leukemia patients with intermediate-risk cytogenetics. Blood. 2011;118:3803-10.

37. Itzykson R, Kosmider O, Cluzeau T, Mansat-De Mas V, Dreyfus $\mathrm{F}$, Beyne-Rauzy O, et al. Impact of TET2 mutations on response rate to azacitidine in myelodysplastic syndromes and low blast count acute myeloid leukemias. Leukemia. 2011;25:1147-52.

38. Mardis ER, Ding L, Dooling DJ, Larson DE, McLellan MD, Chen $\mathrm{K}$, et al. Recurring mutations found by sequencing an acute myeloid leukemia genome. New Engl J Med. 2009;361: 1058-66.

39. Yan H, Parsons DW, Jin G, McLendon R, Rasheed BA, Yuan W, et al. IDH1 and IDH2 mutations in gliomas. New Engl J Med. 2009;360:765-73.

40. Gross S, Cairns RA, Minden MD, Driggers EM, Bittinger MA, Jang HG, et al. Cancer-associated metabolite 2-hydroxyglutarate accumulates in acute myelogenous leukemia with isocitrate dehydrogenase 1 and 2 mutations. J Exp Med. 2010;207:339-44.

41. Ward PS, Patel J, Wise DR, Abdel-Wahab O, Bennett BD, Coller HA, et al. The common feature of leukemia-associated IDH1 and IDH2 mutations is a neomorphic enzyme activity converting alphaketoglutarate to 2-hydroxyglutarate. Cancer Cell. 2010;17:225-34.

42. Parsons DW, Jones S, Zhang X, Lin JC, Leary RJ, Angenendt P, et al. An integrated genomic analysis of human glioblastoma multiforme. Science. 2008;321:1807-12.

43. Patel JP, Gonen M, Figueroa ME, Fernandez H, Sun Z, Racevskis $\mathrm{J}$, et al. Prognostic relevance of integrated genetic profiling in acute myeloid leukemia. New Engl J Med. 2012;366:1079-89.

44. Patnaik MM, Hanson CA, Hodnefield JM, Lasho TL, Finke CM, Knudson RA, et al. Differential prognostic effect of IDH1 versus IDH2 mutations in myelodysplastic syndromes: a Mayo Clinic study of 277 patients. Leukemia. 2012;26:101-5.

45. Tefferi A, Lasho TL, Abdel-Wahab O, Guglielmelli P, Patel J, Caramazza D, et al. IDH1 and IDH2 mutation studies in 1473 patients with chronic-, fibrotic- or blast-phase essential thrombocythemia, polycythemia vera or myelofibrosis. Leukemia. 2010;24:1302-9.

46. Borger DR, Tanabe KK, Fan KC, Lopez HU, Fantin VR, Straley $\mathrm{KS}$, et al. Frequent mutation of isocitrate dehydrogenase (IDH) 1 and IDH2 in cholangiocarcinoma identified through broad-based tumor genotyping. Oncologist. 2012;17:72-9.

47. Amary MF, Bacsi K, Maggiani F, Damato S, Halai D, Berisha F, et al. IDH1 and IDH2 mutations are frequent events in central chondrosarcoma and central and periosteal chondromas but not in other mesenchymal tumours. J Pathol. 2011;224:334-43.

48. Cairns RA, Iqbal J, Lemonnier F, Kucuk C, de Leval L, Jais JP, et al. IDH2 mutations are frequent in angioimmunoblastic T-cell lymphoma. Blood. 2012;119:1901-3.

49. Losman JA, Kaelin WG Jr. What a difference a hydroxyl makes: mutant IDH, (R)-2-hydroxyglutarate, and cancer. Genes Dev. 2013;27:836-52.

50. Figueroa ME, Abdel-Wahab O, Lu C, Ward PS, Patel J, Shih A, et al. Leukemic IDH1 and IDH2 mutations result in a hypermethylation phenotype, disrupt TET2 function, and impair hematopoietic differentiation. Cancer Cell. 2010;18:553-67.

51. Gaidzik VI, Paschka P, Spath D, Habdank M, Kohne CH, Germing U, et al. TET2 mutations in acute myeloid leukemia (AML): results from a comprehensive genetic and clinical analysis of the AML study group. J Clin. 2012;30:1350-7.

52. Dang L, White DW, Gross S, Bennett BD, Bittinger MA, Driggers EM, et al. Cancer-associated IDH1 mutations produce 2-hydroxyglutarate. Nature. 2009;462:739-44.

53. Xu W, Yang H, Liu Y, Yang Y, Wang P, Kim SH, et al. Oncometabolite 2-hydroxyglutarate is a competitive inhibitor of alpha-ketoglutarate-dependent dioxygenases. Cancer Cell. 2011;19:17-30.

54. Losman JA, Looper RE, Koivunen P, Lee S, Schneider RK, McMahon C, et al. (R)-2-hydroxyglutarate is sufficient to promote leukemogenesis and its effects are reversible. Science. 2013;339:1621-5.

55. Chowdhury R, Yeoh KK, Tian YM, Hillringhaus L, Bagg EA, Rose NR, et al. The oncometabolite 2-hydroxyglutarate inhibits histone lysine demethylases. EMBO Rep. 2011;12:463-9.

56. Lu C, Ward PS, Kapoor GS, Rohle D, Turcan S, Abdel-Wahab O, et al. IDH mutation impairs histone demethylation and results in a block to cell differentiation. Nature. 2012;483:474-8.

57. Koivunen P, Lee S, Duncan CG, Lopez G, Lu G, Ramkissoon S, et al. Transformation by the (R)-enantiomer of 2-hydroxyglutarate linked to EGLN activation. Nature. 2012;483:484-8.

58. Sasaki M, Knobbe CB, Munger JC, Lind EF, Brenner D, Brustle A, et al. IDH1(R132H) mutation increases murine haematopoietic progenitors and alters epigenetics. Nature. 2012;488:656-9. 
59. Bralten LB, Kloosterhof NK, Balvers R, Sacchetti A, Lapre L, Lamfers M, et al. IDH1 R132H decreases proliferation of glioma cell lines in vitro and in vivo. Ann Neurol. 2011;69:455-63.

60. Chou WC, Peng KY, Lei WC, Ko BS, Tsay W, Kuo CH, et al. Persistence of mutant isocitrate dehydrogenase in patients with acute myeloid leukemia in remission. Leukemia. 2012;26:527-9.

61. Chou WC, Hou HA, Chen CY, Tang JL, Yao M, Tsay W, et al. Distinct clinical and biologic characteristics in adult acute myeloid leukemia bearing the isocitrate dehydrogenase 1 mutation. Blood. 2010;115:2749-54.

62. Chou WC, Lei WC, Ko BS, Hou HA, Chen CY, Tang JL, et al. The prognostic impact and stability of Isocitrate dehydrogenase 2 mutation in adult patients with acute myeloid leukemia. Leukemia. 2011;25:246-53.

63. Wang F, Travins J, Delabarre B, Penard-Lacronique V, Schalm S, Hansen E, et al. Targeted inhibition of mutant IDH2 in leukemia cells induces cellular differentiation. Science. 2013;340 (6132):622-6.

64. Rohle D, Popovici-Muller J, Palaskas N, Turcan S, Grommes C, Campos C, et al. An inhibitor of mutant IDH1 delays growth and promotes differentiation of glioma cells. Science. 2013; 340(6132):626-30. 